\title{
Mixed-Mode Oscillations in Nonautonomous Modified Chua's Circuit
}

\author{
Ying Ji, Tingting Hua, and Yawei Wang \\ Department of Physics, Faculty of Science, Jiangsu University, Zhenjiang 212013, China \\ Correspondence should be addressed to Yawei Wang; jszjwyw@sina.cn
}

Received 6 April 2015; Revised 23 May 2015; Accepted 26 May 2015

Academic Editor: Gisele Mophou

Copyright (C) 2015 Ying Ji et al. This is an open access article distributed under the Creative Commons Attribution License, which permits unrestricted use, distribution, and reproduction in any medium, provided the original work is properly cited.

A nonautonomous system revised from Chua's circuit with a cubic nonlinearity which could exhibit slow-fast effect is investigated in this paper. Inspired by slow-fast dynamical analysis, the external excitation is considered as a modulation parameter, which changes slowly and periodically. By introducing "transformed phase space," bifurcation analysis of typical periodic mixed-mode oscillations induced by periodic excitation has been described to reveal the associated dynamical mechanisms.

\section{Introduction}

Oscillatory electrical activity called bursting is a common feature of single neuron or neural networks [1]. Bursting behavior, which is also known as mixed-mode oscillation, exhibits a transition between a rest state and a spiking state, owing to a slow variation process modulating fast firing activities. Due to its importance to the information processing in the nervous system, dynamical behavior and classification of types of mixed-mode oscillations (MMOs) have received a considerable attention [2-5].

In the case of experimental research, besides neural firing experiments, electronic circuits are utilized to mimic neurological dynamics. Imitation based on circuits is considered to become a strong choice for implementation and analysis of MMOs due to its high performance [6-8]. It enables prototyping of neural algorithms to test theories of neural computation, structure, network architecture, learning, and plasticity. The simulation of biologically inspired systems in a realtime operation could also be carried out via this approach. This is of particular interest for sensory processing systems and biologically inspired robotics [9]. Meanwhile, it is still relatively difficult to design electronic nonlinear devices capable of reproducing complex oscillations because of the lack of general constructive rules and because of the stability problems related to the dynamical robustness of the circuits [1]. Therefore, the simulation study of oscillator circuits is important to precisely predict the performance.
As for the theoretical study, a series of research revealed the inherent dynamical nature of different MMOs by some crucial bifurcations [10-12]. On the basis of these works, Izhikevich pointed out that the bifurcations are what determines the neuron-computational properties [13]. He considered more comprehensive bifurcations associated with bursting and provided a more complete theoretical classification, that is, "top-down" fast-slow dynamical bifurcation analysis [13]. Neuronal mathematical models with MMOs can be described by a singularly perturbed system of the form:

$$
\begin{aligned}
& \dot{x}=f(x, u) \\
& \dot{u}=\mu g(x, u),
\end{aligned}
$$

where $\mu \ll 1$ represents the radio of fast and slow time scales. The vector $x \in R^{m}$ describes the relatively fast processes associated with the generation of spiking and $u \in R^{k}$ describes relatively slow processes that modulate $x$. There exist two important bifurcations associated with MMOs in fast subsystems (1), that is, the bifurcation of a rest state leading to repetitive spiking and the bifurcation of a repetitive spiking state leading to the rest state. These two important bifurcations represent the interaction between the generation of fast spiking and the dynamics of slow variation, and they can be regarded as an essential basis for the classification of bursting thereby. In addition, there may exist other bifurcations of the rest state or the spiking state, which can commonly lead to 


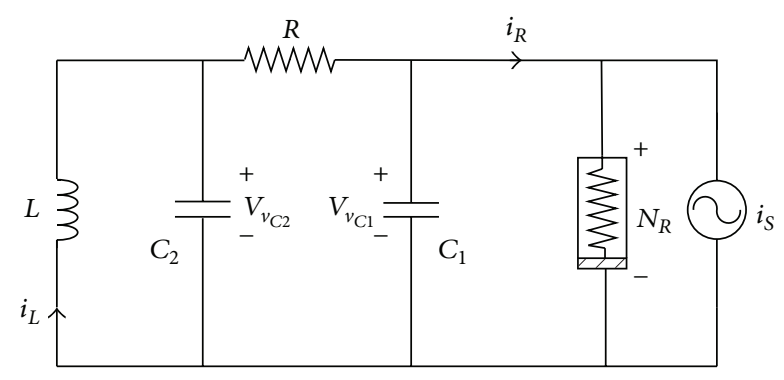

FIgURE 1: Modified Chua's circuit with external current source.

a hysteresis loop and then give rise to different mixed-mode oscillations, that is, the bifurcations from the down-state to the up-state or the opposite case in the fast subsystem with the slow variable $u$ taken as the bifurcation parameter. With this method, the bifurcation mechanism of the bursters has been well summarized by many researchers [14].

However, up to now, most of the work which involves slow-fast effect is confined to the autonomous systems. The MMOs of nonautonomous systems are still the open problems. In this paper, modified Chua's circuit with periodic external excitation is explored. Inspired by Izhikevich's method [13], an analysis of MMOs induced by external excitation is presented. Two different typical bursting phenomena, which depend on whether the spiking state is contained or not, appear in this circuit and the associated bifurcation mechanism is revealed.

\section{The System and the Mixed-Mode Oscillations}

We explore a modified Chua's circuit with periodic external excitation, shown in Figure 1.

In this circuit, the piecewise voltage-current characteristic of the nonlinear resistor is replaced with a cubic nonlinearity. This line of transformation was initiated by Zhong. The advantages of the modification are that it is smooth and thus more suitable for mathematical calculations [15]. The dynamics of the circuit has been described by the following system:

$$
\begin{aligned}
\frac{d v_{C 1}}{d t} & =\frac{\left(v_{C 1}-v_{C 2}\right)}{R C_{1}}-\frac{f\left(v_{C 1}\right)}{C_{1}}+\frac{i_{S}}{C_{1}}, \\
\frac{d v_{C 2}}{d t} & =\frac{\left(v_{C 1}-v_{C 2}\right)}{R C_{2}}+\frac{i_{L}}{C_{2}}, \\
\frac{d i_{L}}{d t} & =-\frac{v_{C 2}}{L},
\end{aligned}
$$

where $v_{C 1}$ and $v_{C 2}$ denote the voltages across the capacitors $C_{1}$ and $C_{2}, i_{L}$ denotes the currents through the inductances $L$, and $i_{S}$ represents the alternating current of the power supply, which could be described as $i_{S}=F \cos \Omega t . f\left(v_{C 1}\right)$ is a cubic nonlinear function.

By employing the transformation $x=v_{C 1}, y=v_{C 2}$, $z=R i_{L}, t=R C_{2} \tau, \alpha=C_{2} / C_{1}, \beta=R C_{2} F / C_{1}$, and $\omega=R C_{2} \Omega$, dimensionless form of system (2) can be written as the following system, where $f(x)=m x+n x^{3}$ :

$$
\begin{aligned}
& \dot{x}=\alpha[y-x-f(x)]+F \cos (\omega \tau) \\
& \dot{y}=x-y+z \\
& \dot{z}=-\beta y .
\end{aligned}
$$

In order to reveal the slow-fast dynamical behaviors of this system, the parameters are fixed at $\beta=15.5, m=-1.25$, $n=0.25, F=1.5$, and $\omega=0.1$, while $\alpha$ is set as a variable. Two typical types of periodic MMOs can be observed in Figure 2 with the variation of the value of $\alpha$; that is, $\alpha=1.0$ and $\alpha=$ 8.0. Meanwhile, there is a common point between these two situations that the combination of large-amplitude oscillation associated with the excitation frequency and the smallamplitude oscillation corresponding to the natural frequency forms the MMOs in the circuit. During each period, which is the same as the period of the external excitation, smallamplitude oscillation locally around two symmetric sets can be observed, while large-amplitude oscillation occurs when the trajectory moves between the small-amplitude oscillations near the two sets, respectively.

It is because there exists an order gap between the excitation frequency and the natural frequency of the system that the dynamics exhibits fast-slow effect (shown in Figure 2). In the following, we will investigate the bifurcation details in the evolution of the dynamics under different conditions.

\section{Dynamical Analysis}

To investigate the mechanism of the MMOs observed in this nonautonomous system, we treat the external excitation as a control function of system (3), which changes periodically and slowly according to the time scale $\omega \tau$. It is denoted by $w=F \cos (\omega \tau)$ for simplicity. Since the value of excitation frequency is much less than that of natural frequency of the system, there are two time scales existing in this system, that is, $\tau$ and $\omega \tau$. It means that $w$ could be regarded as a slow variable, while $x, y$, and $z$ are fast variables. The phase portraits observed in Figures 2(a) and 2(b) are transformed to $w-x-y$ phase space to reveal the modulation effects of the external excitation (shown in Figures 3(a) and 4(a)). That is, $w$ can be treated as a bifurcation parameter of the fast subsystem $(x, y, z)$ according to the slow-fast dynamical bifurcation analysis [13]. Thus, the associated bifurcation diagrams of the fast subsystem can be seen in Figures 3(b) and 4(b) respectively. We now display the dynamical behaviors for these two parameter conditions.

First, we account for MMO for $\alpha=1.0$. The S-shaped curve shown in Figure 3(b) is divided into three branches by fold bifurcation points LP1 and LP2, that is, the solid upper branch, the dotted middle branch, and the solid lower branch. The solid branches represent stable focuses while the dotted branch represents unstable focuses.

To reveal the mechanism of the mixed-mode oscillation, the bifurcation diagram shown in Figure 3(b) is superposed on phase portrait shown in Figure 3(a) in one period (see Figure 3(c)). For the convenience of description, Figure 3(c) 

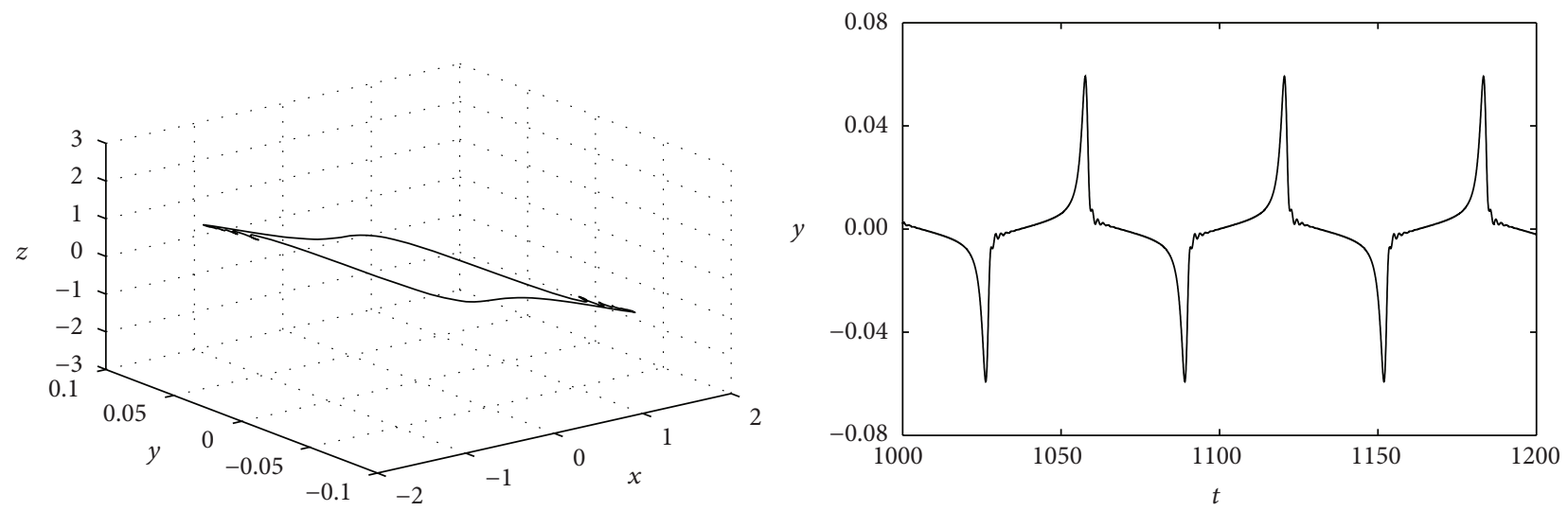

(a)
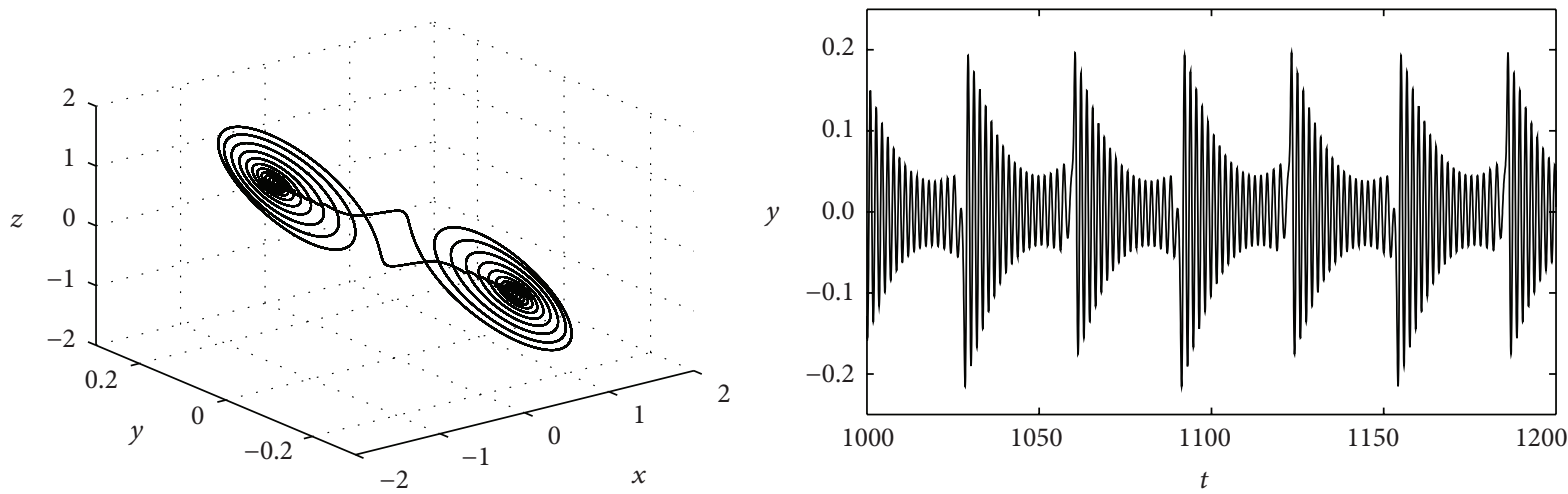

(b)

Figure 2: Two typical types of MMOs observed in system (3) for different parameters. (a) $\alpha=1.0$. (b) $\alpha=8.0$.

is projected onto $w$-x phase plane (seen in Figure 3(d)). The black arrows on the trajectory indicate the direction of the flow.

As shown in Figure 3(d), to the left of point LP1, the lower stable focus point is the only attractor. Thus, when the value of external excitation decreases through the point LP1, the orbit switches to the stable lower branch by the fold bifurcation LP1 (see Figure 3(d)). After reaching the minimum -1.5 , $w$ begins to increase. When $w$ increases through the value of $w_{\mathrm{LP} 2} \approx$ 0.0962, the orbit jumps back to the stable upper branch by means of the fold bifurcation LP2 (see Figure 3(d)). Like that, due to the modulation of the external excitation, the orbit switches between the two stable focuses of the fast subsystem and thus the MMO shown in Figure 3 is created.

It is noted that the bifurcation that involves generation of limit cycle is not observed in the above analysis, which means that there is no spiking state in this case. With the slow variable $w$ decreasing, the upper rest state of the MMO corresponding to the stable focus on the upper branch of the bifurcation curve transits to the lower rest state corresponding to the stable focus on the lower branch via fold bifurcation at LP1. Meanwhile, the lower rest state transits to the upper rest state via fold bifurcation at LP2 with the slow variable $w$ increasing. Thus, a point-point hysteresis loop is generated by the transition between these two rest states, that is, stable "up-state" and "down-state," and the MMO exhibits dynamical behavior of fold/fold point-point hysteresis loop bursting.

As for the case of $\alpha=8.0$, the transformed phase portrait and the bifurcation diagram of system (3) in the $w-x-y$ phase space are shown as Figures 4(a) and 4(b). Similar to the above, the phase portrait and bifurcation diagram are projected onto a phase plane, namely, $w-x$ phase plane (seen in Figures $4(\mathrm{c})$ and $4(\mathrm{~d})$ ) in order to demonstrate the different types of bifurcation clearly. S-shaped curve is divided into three branches by fold bifurcation points LP1 and LP2. On the dotted middle branch, the equilibrium is an unstable focus. On the upper branch, the solid part represents stable focus points while the dotted part represents unstable ones. The stabilities of the focuses change by supercritical Hopf bifurcation and subcritical Hopf bifurcation, which occur at the points H1 and SH1, respectively. Because of the symmetric property of the bifurcation, the case of the lower branch is similar to that of the upper one. The vertical solid lines originated from the points $\mathrm{H} 1$ and $\mathrm{H} 2$ represent stable limit cycles, while the dashed ones originated from the points $\mathrm{SH} 1$ and $\mathrm{SH} 2$ represent unstable limit cycles. As the change of the value of $w$, the unstable limit cycle grows and approaches the stable limit cycle. When the value of $w$ reaches to $w_{\mathrm{LPC1}(2)}$, the two cycles collide and disappear; that is, fold bifurcation of limit cycle occurs, which is shown with LPC1 and LPC2 in Figures 4(b) and $4(\mathrm{~d})$. 


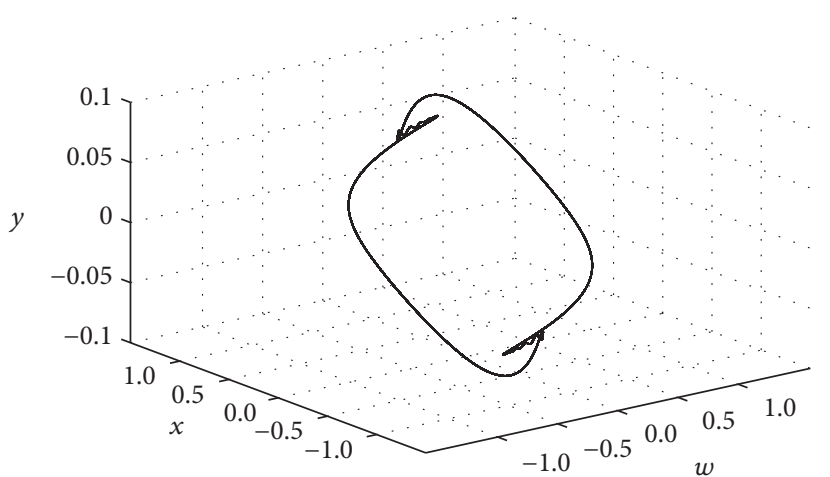

(a)

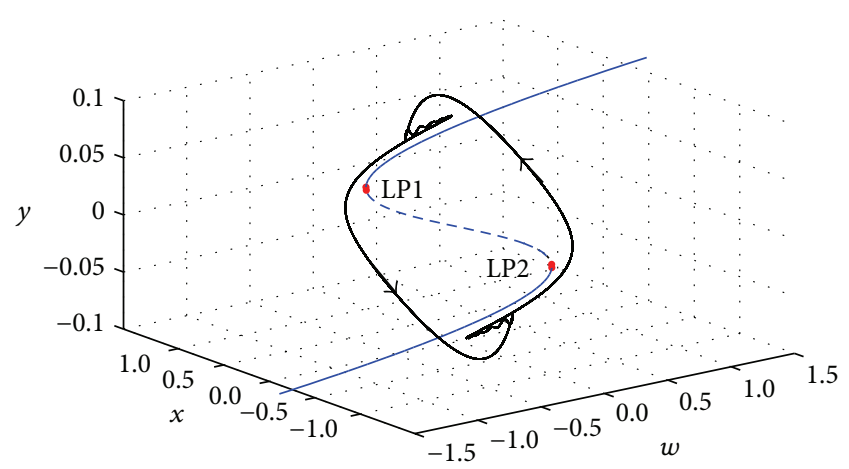

(c)

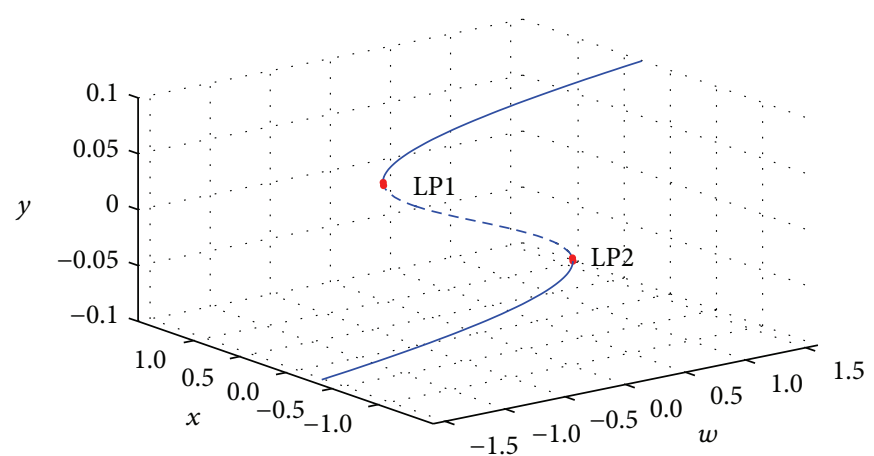

(b)

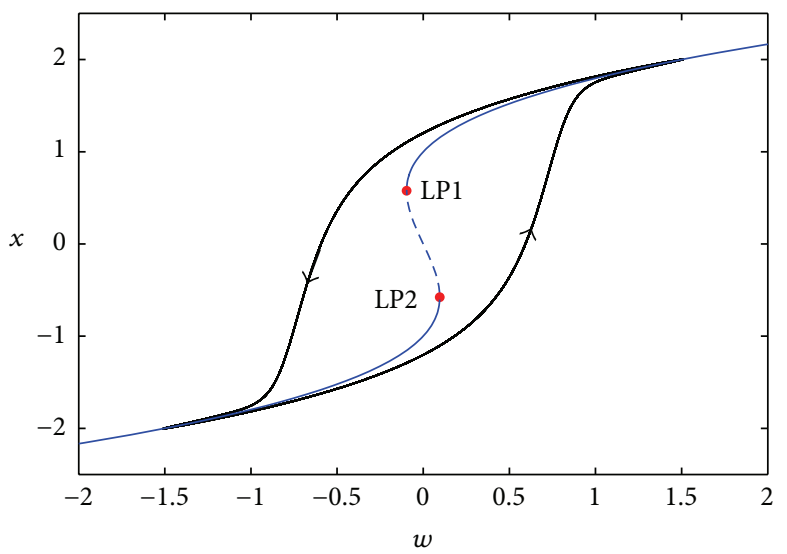

(d)

Figure 3: (a) Transformed phase portrait of system (3) in the $w-x-y$ phase space for $\alpha=1.0$. (b) The bifurcation diagram of system (3) in the $w-x-y$ phase space for $\alpha=1.0$. (c) Symmetric fold/fold point-point hysteresis loop bursting for $\alpha=1.0$. (d) Projection of Figure 3(c) on the $w$-x phase plane. Remarks: solid curve denotes stable equilibrium; dotted curve denotes unstable equilibrium; LP1 and LP2 denote fold bifurcation.

To reveal the mechanism of the mixed-mode oscillation, the bifurcation diagram projected on $w$-x phase plane shown in Figure 4(d) is superposed on the associated phase portrait shown in Figure 4(c) in one period (see Figure 5). The black arrows on the trajectory indicate the direction of the flow.

We will describe one revolution of the oscillation in detail. As shown in Figure 5, to the left of point LP1, the stable limit cycle on the lower branch is the only attractor of the fast subsystem. Thus, beyond that point, the stationary manifold becomes unstable with the decreases of $w$ and the trajectory of the system switches to the stable limit cycle. The repetitive spiking appears by means of fold bifurcation.

With the further decreases of $w$, the trajectory converges to the stable equilibrium by means of fold bifurcation of limit cycle when it passes through LPC2 at $w_{\text {LPC2 }} \approx-1.414$. Noting that $w$ is a periodic function in this letter, the value of $w$ begins to increase after reaching the minimum -1.5 . Thus, the trajectory turns to the right with the increases of $w$ till it arrives at $\mathrm{SH} 2$. As $w$ increases to $w_{\mathrm{SH} 2} \approx-0.839$, subcritical Hopf bifurcation can be found, which leads the equilibrium to lose its stability. And the trajectory begins to oscillate with small amplitude. Till the value of $w$ increases to $w_{\mathrm{H} 2} \approx 0.747$, the orbit withdraws from spiking oscillation by means of supercritical Hopf bifurcation at point $\mathrm{H} 2$ and stable quiescent state appears. Then, with the further increases of $w$, the stable focus on the lower branch loses its stability at point LP2 by fold bifurcation. Then, the trajectory jumps to the upper branch and oscillates around the corresponding limit cycles again.

Because of the symmetric property of the oscillation (seen in Figure 5), the behavior of the orbit at the upper branch is similar to the situation at the lower branch described above. The trajectory passes through the bifurcation points LPC1, SH1, and H1 in turn and then reaches point LP1 to begin another period.

Different from the case of $\alpha=1.0$, spiking state could be observed under the parameter condition with $\alpha=8.0$. Based on the analysis above, it could be found that the quiescent state loses its stability by fold bifurcation, while the spiking state terminates by supercritical Hopf bifurcation. Besides these two bifurcations which are responsible for emergence and termination of the spike state, the bifurcations of hysteresis loop are also considered; that is, fold bifurcation of limit cycle and subcritical Hopf bifurcation occur at LPC1 (LPC2) and $\mathrm{SH} 1 \mathrm{SH} 2)$, respectively, with the slowly changing external excitation that periodically passes through bifurcation 


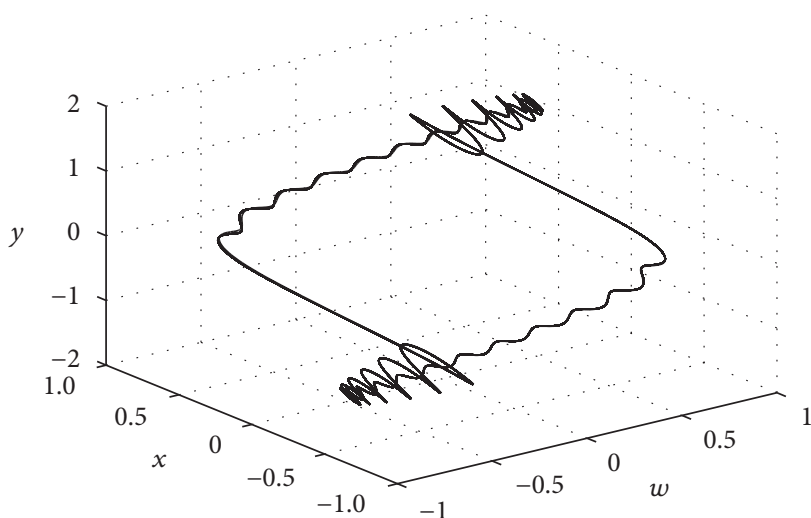

(a)

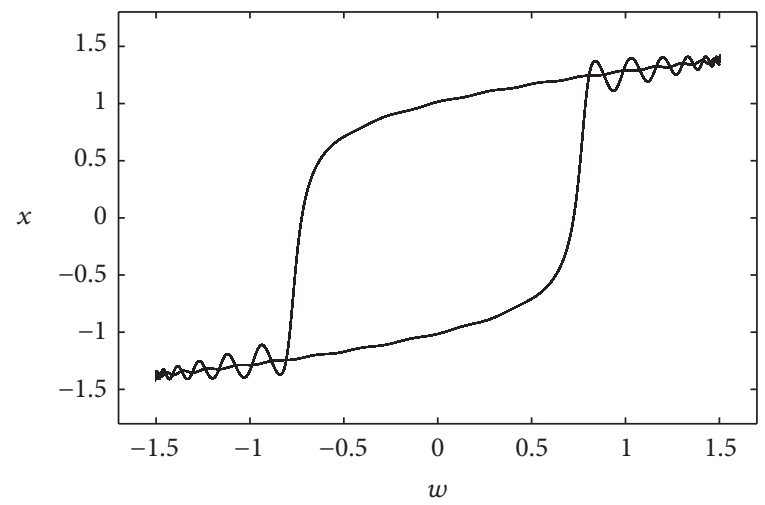

(c)

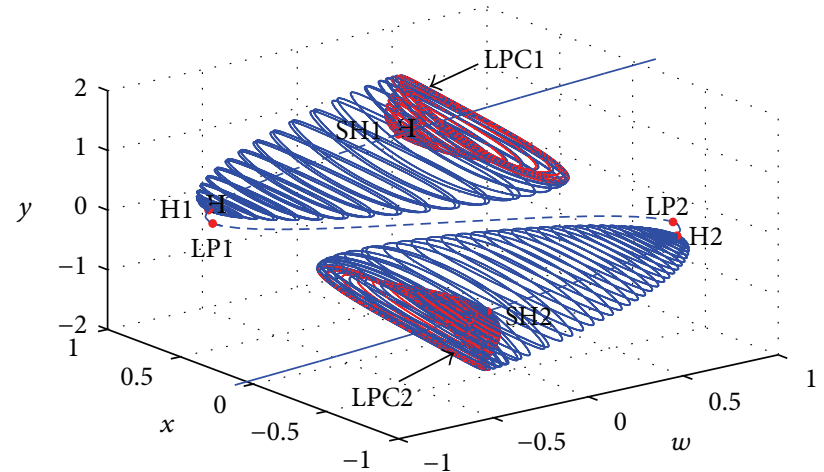

(b)

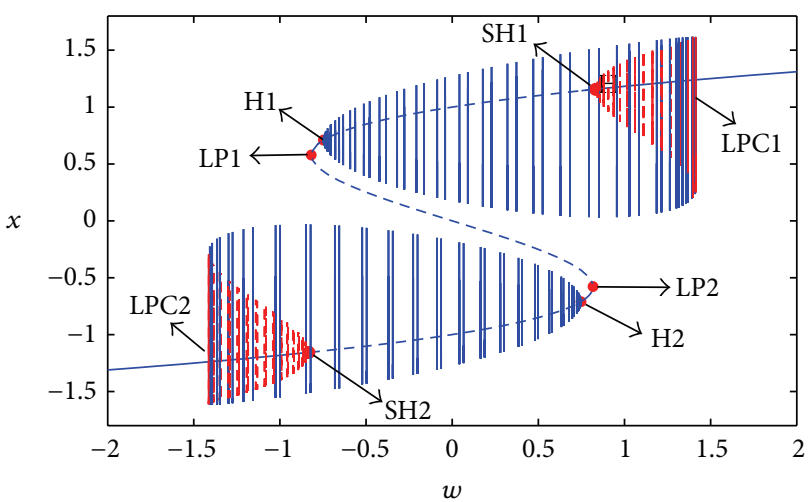

(d)

FIGURE 4: (a) Transformed phase portrait of system (3) in the $w-x-y$ phase space for $\alpha=8.0$. (b) The bifurcation diagram of system (3) in the $w-x-y$ phase space for $\alpha=8.0$. (c) Projection of Figure 4(a) on the $w$-x phase plane. (d) Projection of Figure 4(b) on the $w$ - $x$ phase plane. Remarks: solid curve denotes stable equilibrium; dotted curve denotes unstable equilibrium; LP1 and LP2 denote fold bifurcation; H1 and H2 denote supercritical Hopf bifurcation; SH1 and SH2 denote subcritical Hopf bifurcation; LPC1 and LPC2 denote fold bifurcation of limit cycle; the vertical solid lines denote stable limit cycles; the vertical dashed lines denote unstable limit cycles.

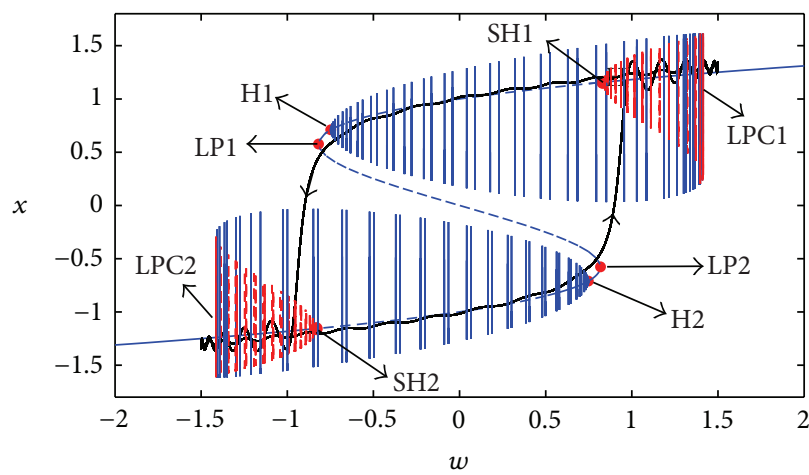

Figure 5: Symmetric sup-Hopf/fold bursting via sub-Hopf/fold-cycle hysteresis loop observed in system (3).

values. Thus, the MMO exhibits dynamical behavior of symmetric sup-Hopf/fold bursting via sub-Hopf/fold-cycle hysteresis loop.

\section{Conclusion}

Mixed-mode oscillations can be observed for the nonautonomous modified Chua's circuit when there exists an order gap between the excitation frequency and the natural frequency of the system. By introducing "transformed phase space," an analysis of this slow-fast effect induced by external excitation has been described to investigate the associated dynamical mechanisms. Two typical cases according to the existence of the spiking state in the oscillation with the slowly varying external excitation are studied in the paper. The first case is symmetric fold/fold point-point hysteresis loop 
bursting, which oscillates without the spiking state. The second one is of symmetric sup-Hopf/fold bursting via subHopf/fold-cycle hysteresis loop. This method is suggested to be applicable to the dynamical systems whose MMOs are induced by external excitation.

\section{Conflict of Interests}

The authors declare that there is no conflict of interests regarding the publication of this paper.

\section{Acknowledgments}

This work is supported by the National Natural Science Foundation of China (Grant nos. 11302086, 11374130, and 11474134), Natural Science Foundation of Jiangsu Province (Grant no. BK20141296), Postdoctoral Science Fund of China (Grant no. 2014M561574), and Postdoctoral Science Fund of Jiangsu Province (Grant no. 1302094B).

\section{References}

[1] G. V. Savino and C. M. Formigli, "Nonlinear electronic circuit with neuron like bursting and spiking dynamics," BioSystems, vol. 97, no. 1, pp. 9-14, 2009.

[2] V. K. Vanag, L. Yang, M. Dolnik, A. M. Zhabotinsky, and I. R. Epstein, "Oscillatory cluster patterns in a homogeneous chemical system with global feedback," Nature, vol. 406, no. 6794, pp. 389-391, 2000.

[3] T. Hayashi, "Mixed-mode oscillations and chaos in a glow discharge," Physical Review Letters, vol. 84, no. 15, pp. 3334-3337, 2000.

[4] F. Zhang, A. Lubbe, Q. Lu, and J. Su, "On bursting solutions near chaotic regimes in a neuron model," Discrete and Continuous Dynamical Systems, Series S, vol. 7, no. 6, pp. 1363-1383, 2014.

[5] W. Marszalek and Z. Trzaska, "Memristive circuits with steadystate mixed-mode oscillations," Electronics Letters, vol. 50, no. 18, pp. 1275-1277, 2014.

[6] T. M. Massoud and T. K. Horiuchi, "A neuromorphic VLSI head direction cell system," IEEE Transactions on Circuits and Systems. I. Regular Papers, vol. 58, no. 1, pp. 150-163, 2011.

[7] A. Basu, S. Ramakrishnan, C. Petre, S. Koziol, S. Brink, and P. E. Hasler, "Neural dynamics in reconfigurable silicon," IEEE Transactions on Biomedical Circuits and Systems, vol. 4, no. 5, pp. 311-319, 2010.

[8] J. V. Arthur and K. A. Boahen, "Silicon-neuron design: a dynamical systems approach," IEEE Transactions on Circuits and Systems. I. Regular Papers, vol. 58, no. 5, pp. 1034-1043, 2011.

[9] H. Soleimani, A. Ahmadi, and M. Bavandpour, "Biologically inspired spiking neurons: piecewise linear models and digital implementation," IEEE Transactions on Circuits and Systems I: Regular Papers, vol. 59, no. 12, pp. 2991-3004, 2012.

[10] J. Rinzel, "Bursting oscillations in an excitable membrane model," in Ordinary and Partial Differential Equations, B. D. Sleeman and R. J. Jarvis, Eds., vol. 1151 of Lecture Notes in Mathematics, pp. 304-316, Springer, Berlin, Germany, 1985.

[11] A. Sherman and J. Rinzel, "Rhythmogenic effects of weak electrotonic coupling in neuronal models," Proceedings of the National Academy of Sciences of the United States of America, vol. 89, no. 6, pp. 2471-2474, 1992.
[12] P. Smolen, D. Terman, and J. Rinzel, "Properties of a bursting model with two slow inhibitory variables," SIAM Journal on Applied Mathematics, vol. 53, no. 3, pp. 861-892, 1993.

[13] E. M. Izhikevich, "Neural excitability, spiking and bursting," International Journal of Bifurcation and Chaos in Applied Sciences and Engineering, vol. 10, no. 6, pp. 1171-1266, 2000.

[14] Z. Q. Yang and Q. S. Lu, "Different types of bursting in Chay neuronal model," Science in China, Series G: Physics, Mechanics and Astronomy, vol. 51, no. 6, pp. 687-698, 2008.

[15] G.-Q. Zhong, "Implementation of Chua's circuit with a cubic nonlinearity," IEEE Transactions on Circuits and Systems I: Fundamental Theory and Applications, vol. 41, no. 12, pp. 934941, 1994. 


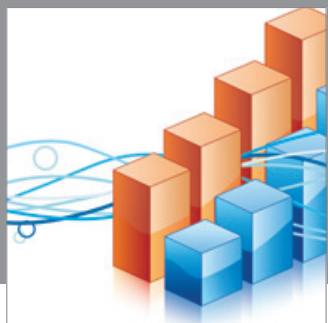

Advances in

Operations Research

mansans

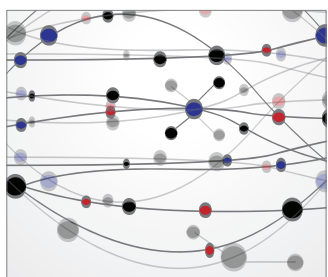

The Scientific World Journal
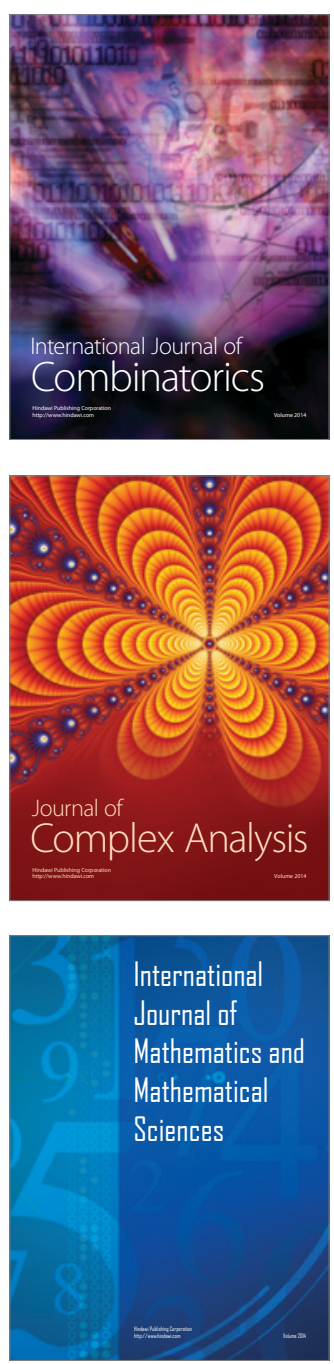
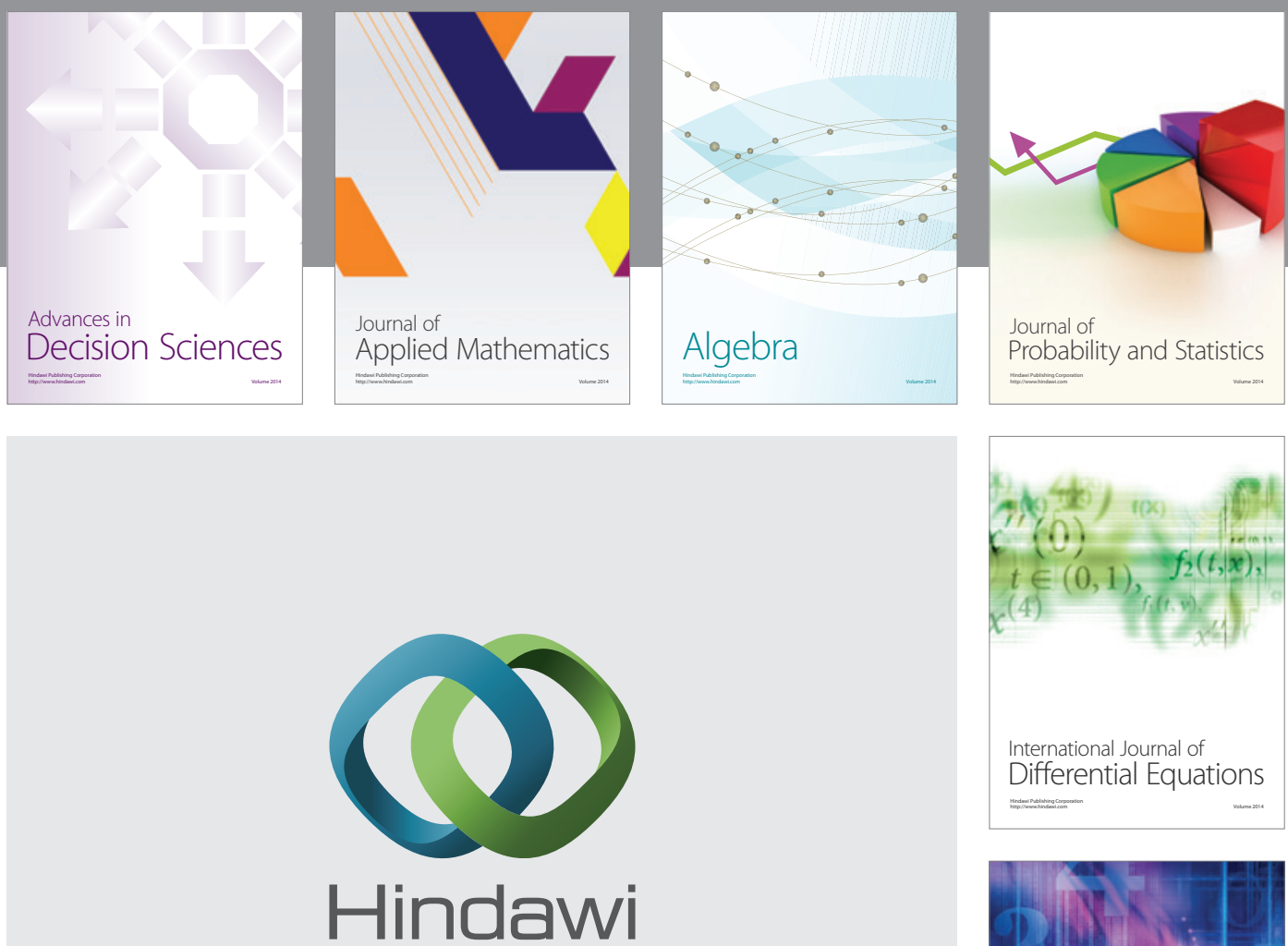

Submit your manuscripts at http://www.hindawi.com
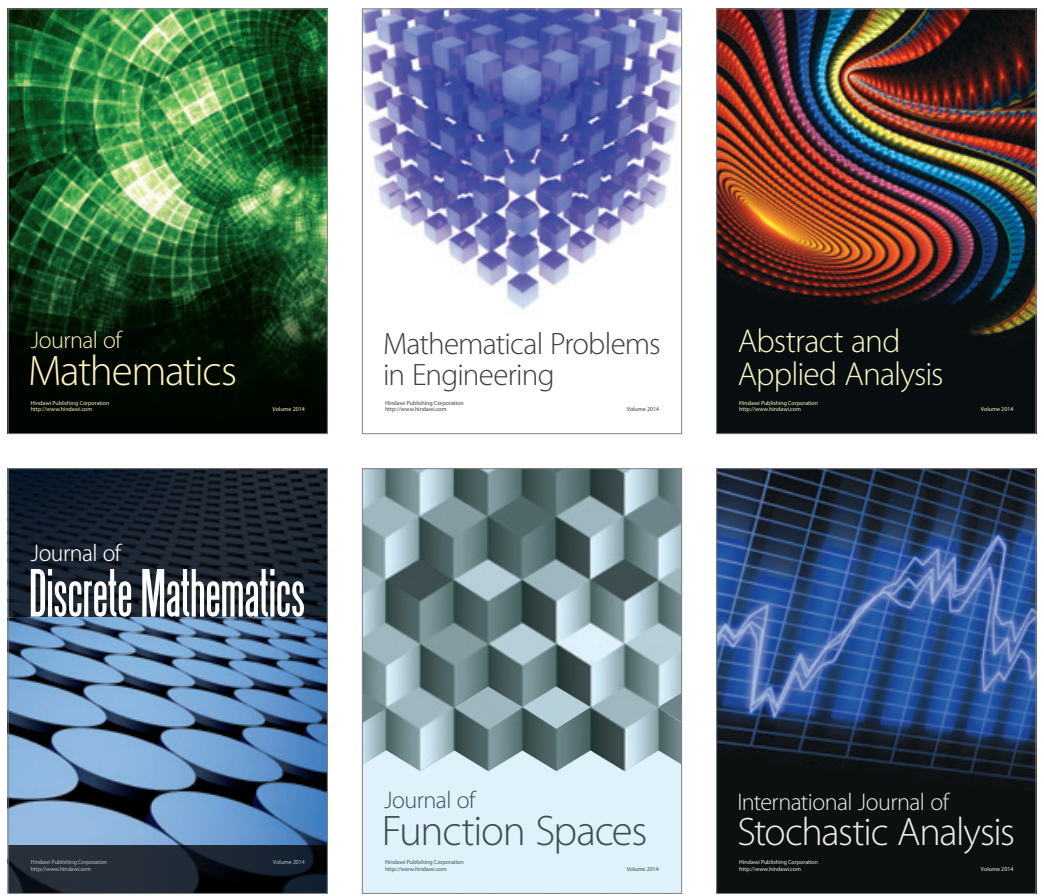

Journal of

Function Spaces

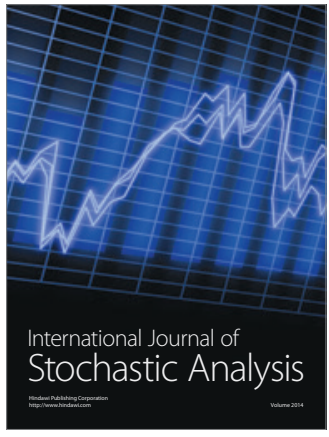

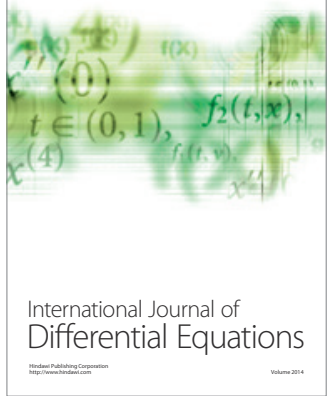
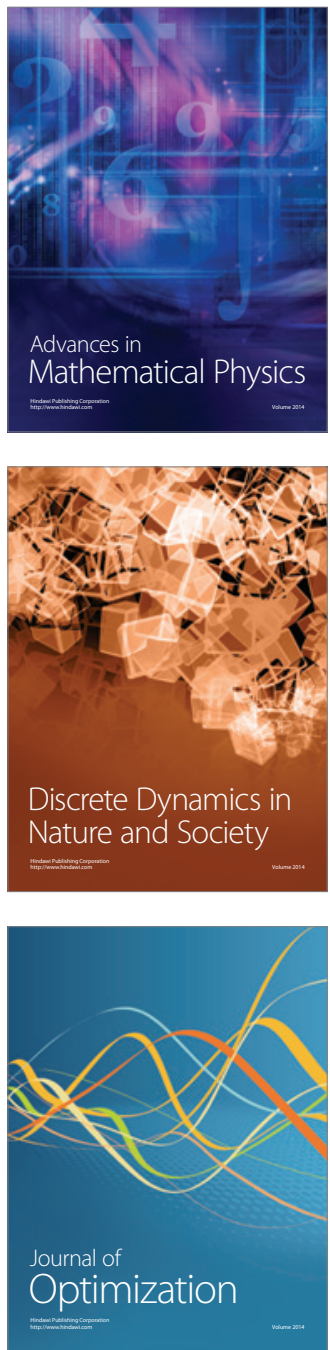